\title{
A Sudden Change of Heart: A Case of Rapidly Reversed Stress Cardiomyopathy in a Critically III Patient
}

\author{
Michael I. Brener ${ }^{\mathrm{a}, \mathrm{c}}$, Ali R. Keramati ${ }^{\mathrm{a}}$, Marek A. Mirski ${ }^{\mathrm{b}}$, and Oscar H. Cingolani ${ }^{\mathrm{a}}$
}

\begin{abstract}
We report the case of a 79-year-old woman who presented to our hospital for elective removal of an infratentorial meningioma and suffered a periprocedural cardiac arrest. Shortly after uncomplicated induction of anesthesia prior to the surgery, the patient became hypotensive and bradycardic, culminating ultimately in a cardiac arrest with pulseless electrical activity. Return of spontaneous circulation occurred within 90 seconds of arrest, but the patient remained dependent on maximal doses of epinephrine and dopamine for hemodynamic support. Echocardiography performed on the day of cardiac arrest revealed a newly depressed left ventricular ejection fraction (LVEF) of $15-20 \%$ with an apical ballooning pattern. Left heart catheterization showed no obstructive coronary lesions to explain her depressed ejection fraction. A diagnosis of stress cardiomyopathy (SCM) was made given the echocardiographic findings and absence of concomitant coronary disease. Within the next 24 hours, the patient was liberated from inotropic support, and at 6-month follow-up, her LVEF returned to 55\% and she had no heart failure symptoms.
\end{abstract}

Keywords: Stress cardiomyopathy; Cardiac arrest; Catecholamines

\section{Introduction}

Stress cardiomyopathy (SCM), a disease with many names like the "broken heart syndrome", Takotsubo cardiomyopathy, and apical ballooning syndrome, is characterized by regional myocardial dysfunction, typically occurring in the wake of a significant physical or emotional stressor. The pathophysiology of the condition remains incompletely understood, yet the

Manuscript accepted for publication June 08, 2016

aDepartment of Medicine and Cardiology, Johns Hopkins Hospital, Baltimore, MD, USA

bepartment of Anesthesiology and Critical Care Medicine, Johns Hopkins Hospital, Baltimore, MD, USA

${ }^{\mathrm{c} C o r r e s p o n d i n g ~ A u t h o r: ~ M i c h a e l ~ I . ~ B r e n e r, ~ D e p a r t m e n t ~ o f ~ M e d i c i n e ~ a n d ~ C a r-~}$ diology, Johns Hopkins Hospital, 600 North Wolfe Street, Harvey Building, Room 808, Baltimore, MD 21287, USA. Email: michael.brener@jhmi.edu

doi: http://dx.doi.org/10.14740/cr471w effects of catecholamines on select portions of myocardium are thought to play an integral role. The degree of cardiac dysfunction in this condition is variable, and in the largest contemporary registry of patients with SCM, only $9.9 \%$ developed cardiogenic shock [1]. Although uncommon, this severe presentation of the disease is critical to appreciate, and we present a case of the disease at its extreme, with a patient in cardiogenic shock.

\section{Case Report}

A 79-year-old woman with a history of an infratentorial meningioma was admitted to the coronary care unit in cardiogenic shock. The morning of admission, she was brought to the operating room for elective meningioma resection. Her past medical history was significant only for modest essential hypertension and no known coronary ischemia or dysrhythmias. Following routine induction of anesthesia with securement of the airway and institution of mechanical ventilation, sinus bradycardia associated with profound hypotension $(70 / 40 \mathrm{~mm} \mathrm{Hg})$ ensued, and this hemodynamic perturbation subsequently progressed to cardiac standstill with absence of peripheral pulses. Cardiopulmonary resuscitation (CPR) was initiated and return of spontaneous circulation (ROSC) was achieved after $90 \mathrm{~s}$ with one round of CPR and $1 \mathrm{mg}$ of intravenous epinephrine. After ROSC, her vital signs were notable for sinus bradycardia with a systolic blood pressure initially between 140 and $170 \mathrm{~mm}$ $\mathrm{Hg}$, which quickly declined to less than $90 \mathrm{~mm} \mathrm{Hg}$ despite continuous infusions of high-dose norepinephrine $(3 \mu \mathrm{g} / \mathrm{kg} / \mathrm{min})$ and dopamine $(20 \mu \mathrm{g} / \mathrm{kg} / \mathrm{min})$. The surface electrocardiogram post-ROSC revealed new T-wave inversions in the precordial leads. Intra-operative transthoracic echocardiography shortly after cardiac arrest demonstrated a left ventricular ejection fraction (LVEF) of $15-20 \%$ with severely hypokinetic mid and distal segments and a hyperkinetic left ventricular base consistent with SCM (Fig. 1 and Supplementary Video 1, www. cardiologyres.org). In the setting of her continued hemodynamic instability, left heart catheterization was performed and an intra-aortic balloon pump was placed. The coronary anatomy was notable for only mild, non-obstructive disease (Fig. 2).

In the subsequent $6 \mathrm{~h}$, her hemodynamics improved markedly with supportive care. Serum troponin I levels peaked at $1.67 \mathrm{ng} / \mathrm{mL}$ and a newly prolonged QT interval developed (Fig. 


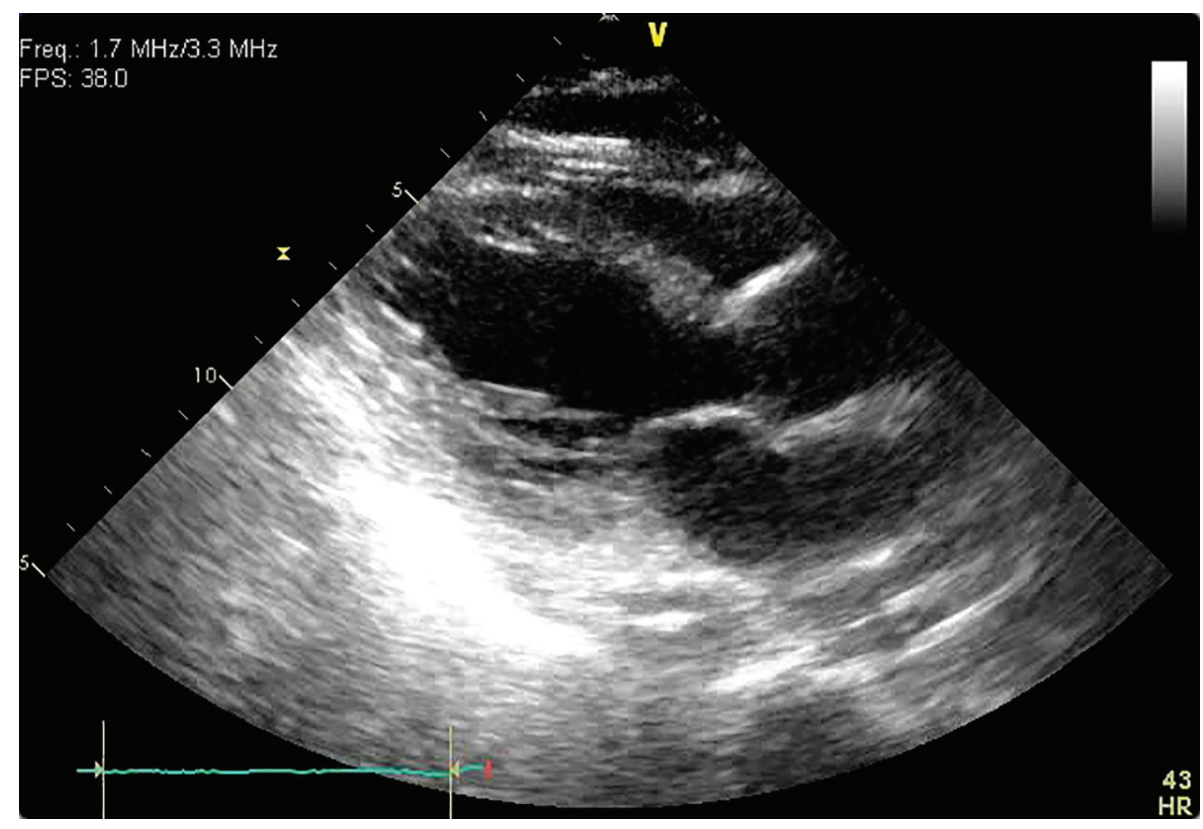

Figure 1. Parasternal long axis view during systole. Ballooning of the apical segments of the left ventricle is apparent.

3). Inotropic and mechanical supports were rapidly weaned off over the following $6 \mathrm{~h}$ and she was successfully extubated. Once awake, she denied any preceding emotional stressors. Repeat echocardiography $12 \mathrm{~h}$ after the initial study revealed a LVEF of $75-80 \%$, near cavity obliteration during systole, and no regional wall motion abnormalities (Supplementary Video 2, www.cardiologyres.org). At 6-month follow-up, the patient denied any symptoms of heart failure and her EF normalized to $55 \%$.

\section{Discussion}

This case highlights many of the classic elements of SCM: a post-menopausal female with a primary neurologic disease; a temporal correlation with significant stress and exposure to catecholamines; prolongation of the QT interval; low-level cardiac enzyme elevations; and reversible, often transient left ventricular dysfunction, which at the extreme, results in cardiogenic shock. Although reversal of left ventricular dysfunction is the norm in SCM, the time course of this patient's improvement is remarkable. Most studies of SCM cite echocardiographic and symptom improvement occurring within days to weeks of diagnosis, and to our knowledge, the earliest reported echocardiographic resolution occurred 5 days following diagnosis in these case series $[2,3]$.

SCM has been associated with administration of catecholamines (i.e. dobutamine during stress testing or epinephrine for treatment of anaphylaxis), and has occurred in the wake of CPR, which we believe occurred in this case [4]. From a pathophysiological standpoint, a catecholamine surge, iatrogenic or otherwise, can impair myocyte contractility, particularly at the apex where the highest concentrations of adrenergic receptors are localized [5].

In summary, we describe a case of transient, albeit severe SCM that reversed rapidly. To our knowledge, this is the fast-

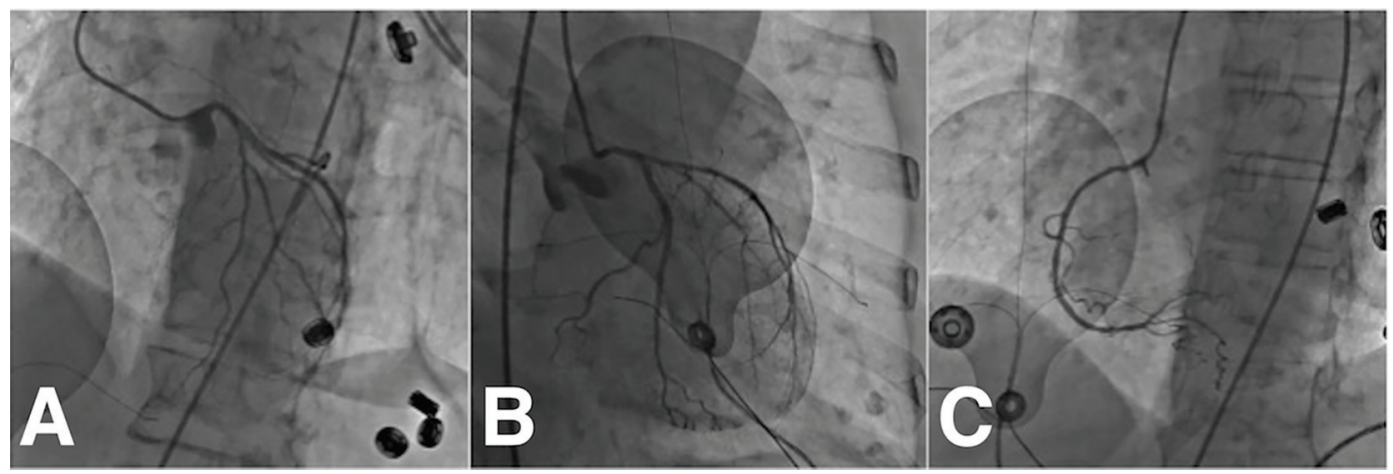

Figure 2. Post-arrest coronary angiogram. There is right dominant circulation. The coronary arteries are diffusely constricted. The left main is angiographically normal. The left anterior descending artery has minimal disease (A). The left circumflex has an ostial $30 \%$ stenosis (B). The right coronary artery has minimal disease, and supplies the posterior descending artery (C). 


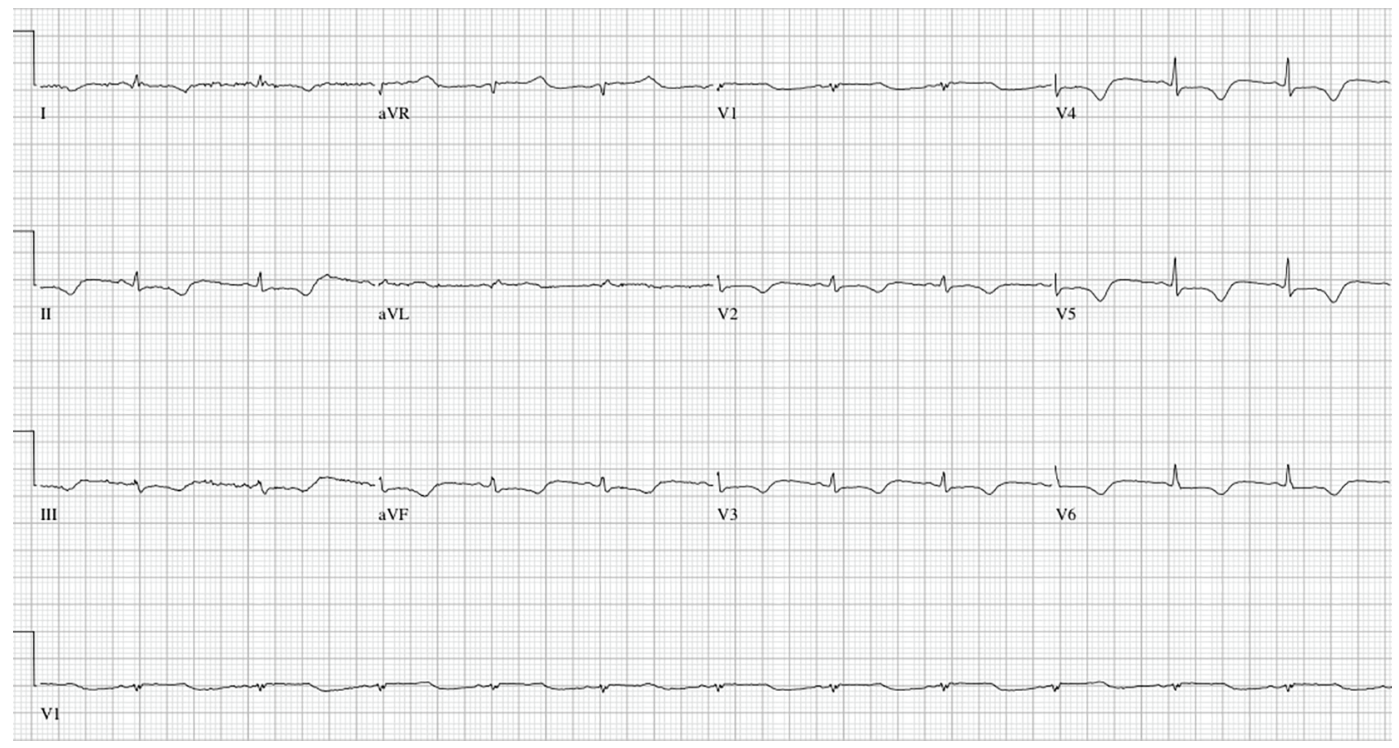

Figure 3. ECG taken $12 \mathrm{~h}$ after cardiac arrest. Note the low voltages, inferior and lateral precordial lead ST segment depressions, and the prolonged QT interval.

est recovery ever reported. This case highlights the role of catecholaminergic excess, one of the proposed mechanisms underlying the disease's pathophysiology, and also reinforces the need of aggressive supportive therapy early in the disease.

\section{Funding Sources}

None.

\section{Conflicts of Interest}

The authors declare that there are no conflicts of interest regarding the publication of this paper.

\section{References}

1. Templin C, Ghadri JR, Diekmann J, Napp LC, Bataiosu
DR, Jaguszewski M, Cammann VL, et al. Clinical Features and Outcomes of Takotsubo (Stress) Cardiomyopathy. N Engl J Med. 2015;373(10):929-938.

2. Wittstein IS, Thiemann DR, Lima JA, Baughman KL, Schulman SP, Gerstenblith G, Wu KC, et al. Neurohumoral features of myocardial stunning due to sudden emotional stress. N Engl J Med. 2005;352(6):539-548.

3. Sharkey SW, Lesser JR, Zenovich AG, Maron MS, Lindberg J, Longe TF, Maron BJ. Acute and reversible cardiomyopathy provoked by stress in women from the United States. Circulation. 2005;111(4):472-479.

4. Copetti R, Peric D, Amore G, Guglielmo N, Federici N, Cominotto F. Transient Tako-Tsubo cardiomyopathy after cardiopulmonary resuscitation: a causal role of adrenaline? Resuscitation. 2013;84(2):e45-46.

5. Litvinov IV, Kotowycz MA, Wassmann S. Iatrogenic epinephrine-induced reverse Takotsubo cardiomyopathy: direct evidence supporting the role of catecholamines in the pathophysiology of the "broken heart syndrome". Clin Res Cardiol. 2009;98(7):457-462. 